\title{
Color space and color channel selection on image segmentation of food images
}

\author{
Luthfi Maulana a, Yusuf Gladiensyah Bihanda b, Yuita Arum Sari c \\ $a, b, c$ Department of Information Engineering, Universitas Brawijaya, Malang, Indonesia \\ email:aluthfi_m@student.ub.ac.id,bchanyusufsj@student.ub.ac.id,cyuita@ub.ac.id
}

\begin{tabular}{l}
\hline A R T I C L E I N F O \\
\hline Article history: \\
Received 20 July 2020 \\
Revised 31 August 2020 \\
Accepted 31 August 2020 \\
Published 1 September 2020 \\
\hline $\begin{array}{l}\text { Keywords: } \\
\text { color channel } \\
\text { color space } \\
\text { food image segmentation } \\
\text { food images } \\
\text { image segmentation }\end{array}$ \\
\hline
\end{tabular}

IEEE style in citing this article:

L. Maulana, Y. G. Bihanda and Y. A. Sari, "Color space and color channel selection on image segmentation of food images," Register: Jurnal Ilmiah Teknologi Sistem Informasi, vol. 6, no. 2, pp. $141-151,2020$

\begin{abstract}
A B S T R A C T
Image segmentation is a predefined process of image processing to determine a specific object. One of the problems in food recognition and food estimation is the lack of quality of the result of image segmentation. This paper presents a comparative study of different color space and color channel selection in image segmentation of food images. Based on previous research regarding image segmentation used in food leftover estimation, this paper proposed a different approach to selecting color space and color channel based on the score of Intersection Over Union (IOU) and Dice from the whole dataset. The color transformation is required, and five color spaces were used: CIELAB, HSV, YUV, $\mathrm{YCbCr}$, and HLS. The result shows that $A$ in LAB and $H$ in HLS are better to produce segmentation than other color channels, with the Dice score of both is 5 (the highest score). It concludes that this color channel selection is applicable to be embedded in the Automatic Food Leftover Estimation (AFLE) algorithm.
\end{abstract}

2020 Register: Jurnal IImiah Teknologi Sistem Informasi (Scientific Journal of Information System Technology) with CC BY NC SA license.

\section{Introduction}

Image segmentation is the first stage in many applications of food recognition or measuring weight by food images. This research is proliferating since those applications can be applied for dietary assessment $[1,2,3]$. Besides, it also measures the weight of food images [4, 5], to classify food images [6, 7], food image retrieval $[8,9,10]$ and to acknowledge the area of food and non-food [11].

Color space and color channel selection are two critical aspects of image segmentation, with both determining the result of image segmentation. In the preliminary research, when applying the Automatic Food Leftover Estimation (AFLE) algorithm, in which image segmentation was used to estimate food leftover weights, having problems in the segmentation process. The beginning step of segmentation is by transforming RGB to another color space, which is CIELAB was implemented instead of RGB [5, 12]. Taking a case from [5], it used color space with B color channel selection to determine the area of food image in the compartment. The AFLE algorithm has constant 'cd' that depends on user input is based on the quality of the "container-d," which is the best segmentation result out of three other containers. The quality of image segmentation alters to weight estimation as well.

Although the result from previous research is acceptable, with many more color spaces and color channels that can be utilized, there is a possibility that other color space and color channel can give a better result than previous research results. In [13], state that there is no single "best" color space that 
works for every image so that evaluation is needed to determine which color space is best used on a particular image. In this work, an image segmentation treated using different color space and color channels to determine which color space and color channel that best for food images in the compartment of the tray box image, using "Fried Rice with Egg and Cucumber" menu for the test image.

Because there are no color space and color channel recommended for testing, five different color spaces are used: CIELAB, HSV, HSL, YCbCr, and YUV. Different color spaces can influence the quality of image representation based on [14]. For the metrics to test the segmented images' quality, Intersection Over Union (IOU) and Dice Score [15]. IOU is chosen based on the advantage in considering the proportion of the properly assigned pixels and penalizing false positive [16]. Dice score is also used as a metrics accompanying IOU for the reason that IOU and Dice's score is a great metrics to use, although not interchangeably [17].

\section{Material and Methods}

\subsection{Dataset}

There are five fried rice images with egg and cucumber in a tray box taken using a webcam, with each image differing in the size of content and lighting. The dataset contains three images of real food leftover, an image with a full food item, and a mock food leftover [1]. Each image on the dataset is segmented according to the method, as explained in the following subsection. We used tray box images with both before eaten and after being eaten in each compartment. We used a white tray and captured it in a perpendicular position with our prototype, Smart Nutrition Box (SNB). We focus on image segmentation's quality when recognizing the food area in each compartment of the tray box. So, the first stage is cropping the whole tray box area into compartments, as shown in Figure 1. The cropping step was processed manually by identifying the coordinates of each compartment and the size of it. After the cropping process, image segmentation is applied based on the color channel in each color space. The result in total 200 images for each compartment for the dataset.
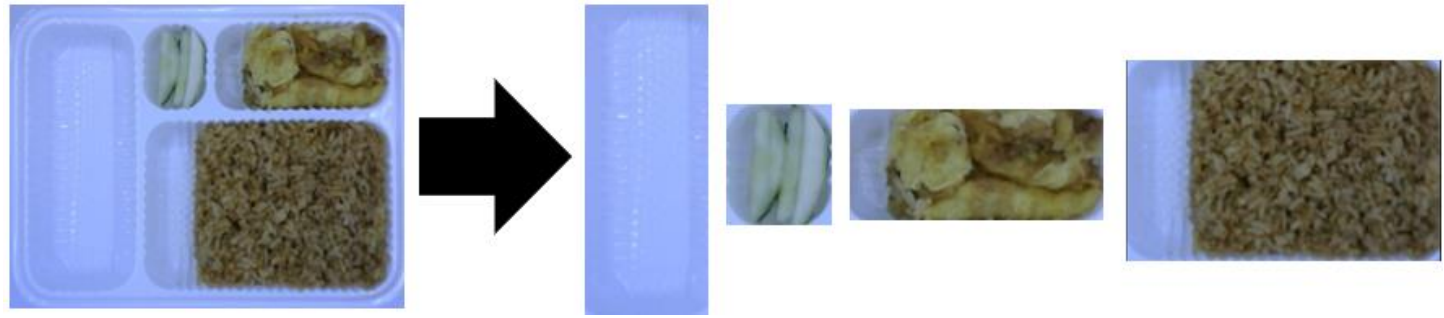

Figure 1. Cropping step

\subsection{Image segmentation}

This paper's image segmentation is the Otsu algorithm, combined with histogram equalization and filtering using Median Filtering and Gaussian Filtering, as depicted in Figure 2. Before computing employing Histogram Equalization, the conversion of color space is from default color space, RGB.

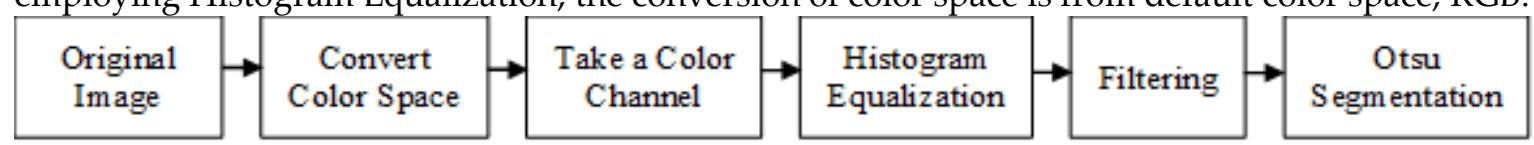

Figure 2. Image segmentation method

In this research, each segmentation is only using one color channel for each color space used. The segmentation may lead to some redundancy because some color space is sharing the same color channel. In the end, it leads to 10 color channels tested, including CIELAB. There are ten color channels used in this research: $H$ of HLS, $L$ of HLS, $S$ of HLS, $V$ of HSV, $A$ of LAB, $B$ of LAB, $\mathrm{Cb}$ of $Y C b C r, C r$ of $Y C b C r$, $\mathrm{Y}$ of $\mathrm{YCbCr}$, and $\mathrm{U}$ of $\mathrm{YUV}$. So, by using ten color channels used in this research, each image will yield 40 data, totaling 200 data of food images in the compartment.

\subsection{Color transformation of color spaces}

RGB is a color space that is not a uniform and dependent device if taken on different devices produces different RGB values. Image processing or computer vision color transformation is necessary for some applications since it can affect the feature extraction process. The different color spaces deliver different perceptions in the color model. For instance, when we apply RGB, we do not need to distinguish color 
components with its lightness component, but it different when we apply other color spaces. The following subsection explains other color spaces that be used in the experiment of this paper.

\section{CIELAB (LAB)}

$\mathrm{LAB}$ is a color space that can be used for images with different lighting conditions and close to human vision interpretation. Therefore, this research requires the transformation of color space under the representation of the human eye. The transformation of color space from RGB to LAB is shown by the following process (Equation 1-5). In this paper, we utilize the $A$ and $B$ color channel since, in the previous research, $A$ [18] dan $B$ [19] color channel has good segmentation results for handling food images in the white plate boundary shape.

$\left[\begin{array}{l}R \\ G \\ B\end{array}\right]\left[\begin{array}{ccc}0.481847 & -0.15866 & -0.082835 \\ -0.001160 & 0.25243 & 0.015708 \\ 0.00002000-0.0025498 & 0.17860\end{array}\right]=\left[\begin{array}{l}X \\ Y \\ Z\end{array}\right]$

$L=\left\{\begin{array}{c}116\left(\frac{Y}{Y_{n}}\right)-16, \frac{Y}{Y_{n}}>0.00856 \\ 903.3\left(\frac{Y}{Y_{n}}\right)\end{array}\right.$

$A=500\left(f\left(\frac{X}{X_{n}}\right)-f\left(\frac{Y}{Y_{n}}\right)\right)$

$B=500\left(f\left(\frac{Y}{Y_{n}}\right)-f\left(\frac{Z}{Z_{n}}\right)\right)$

$f(s)= \begin{cases}S^{\frac{1}{3}}, & s>0.008856 \\ 7.787(s)+\left(\frac{16}{116}\right), & s \leq 0.008856\end{cases}$

In RGB to LAB conversion, the RGB color space is converted to $\mathrm{XYZ}$ color space. The conversion is calculated using matrix multiplication with a constant matrix. The detail of the matrix multiplication following (Equation 1).

After RGB to $\mathrm{XYZ}$ conversion, the $\mathrm{XYZ}$ color space is converted to LAB color space. For the conversion, reference white constants $\left(X_{n}, Y_{n}, Z_{n}\right)$ are required. The value of $L$ channel calculated by using the value of $Y$ divided by $Y_{n}$ multiplied by a constant, or multiplied and subtracted by a constant depending on the $Y$ divided by $Y_{n}$ value. The $A$ channel calculated by 500 multiplied with the value of $f$ with $X$ divided by $X n$ minus $f$ with $Y$ divided by $Y_{n}$. The $A$ channel calculated by 500 multiplied with the value of $f$ with $\mathrm{Y}$ divided by $\mathrm{Y}_{\mathrm{n}}$ minus $f$ with $\mathrm{Z}$ divided by $\mathrm{Z}_{\mathrm{n}}$. Both of $A$ and $B$ calculation used a function $f$, which mapped into either the variable to the power of $1 / 3$ or the variable multiplied then added wit some constants, depending on the value of the variable.
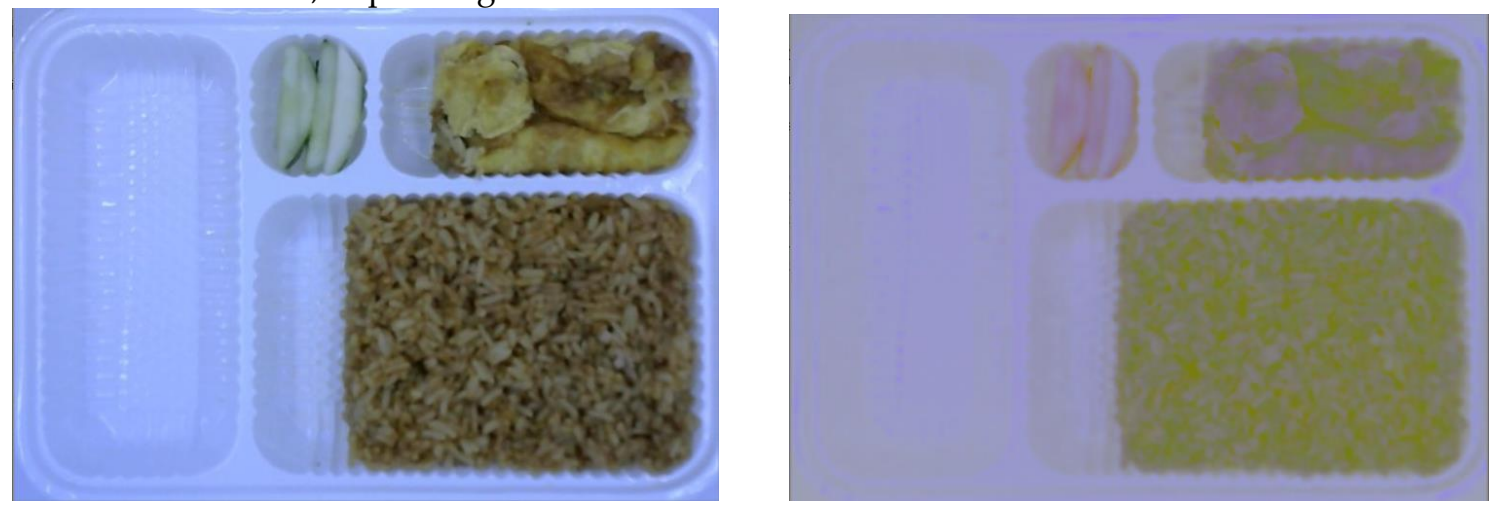

Figure 3. Image comparison between original image with LAB converted image

Hue Saturation Value (HSV)

Hue Saturation Value (HSV) are two alternative representations of the RGB color model. Color space represents the color, as seen by the human eye. HSV characterizes colors based on hue, Saturation while the color channel value describes the level of brightness (brightness). In order to convert RGB images to HSV color space, the following Equation is calculated as stated in Equation 6. In this paper, we apply only a single channel of HSV to 
further observation as a comparison, that is $\mathrm{V}$ based on the previous experiment in [11] since the segmentation of food image in the first round of segmentation was good by involving $V$ color channel combined Gaussian blur with Otsu thresholding.

In RGB to HSV conversion, the $V$ channel computed first, with $V$ equals to the maximum value of each RGB channel. Next, $S$ channel computed by $V$ subtracted from the result of minimum value of each RGB channel divided by $V$. The $H$ channel computed according to the $V$ channel value. If $V$ value equal to $R$ value, then $H$ channel equals to the result of $G$ subtracted by $B$ divided by $S$ multiplied by six. If $V$ value equal to $G$ value, then $H$ channel equals to the result of $B$ subtracted from $R$ divided by $S$ multiplied by six, then summed to a constant $(1 / 3)$. If $V$ value equal to $B$ value, then $H$ channel equals to the result of $R$ subtracted by $G$ divided with $S$, then summed to a constant (2/3).

$V=\max (R, G, B)$

$S=V-\frac{\min (R, G, B)}{V}$

$H=\frac{G-B}{6 S}$, if $V=R$

$H=\frac{1}{3}+\frac{B-R}{6 S}$, if $V=G$

$H=\frac{2}{3}+\frac{R-G}{S}$, if $V=B$
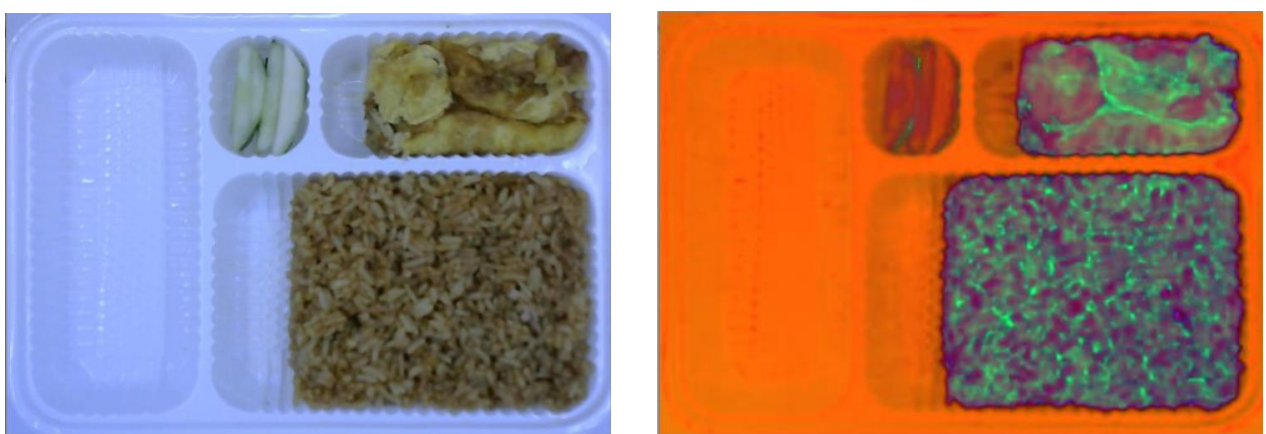

Figure 4. Image comparison between original image with HSV converted image

HSL

HSL color space consists of Hue $(H)$, Saturation $(S)$, and Lightness $(L)$, which is quite similar to HSV. The conversion formula of RGB to HSL is given in the Equation 7-10. This paper implements those three color channels to improve the segmentation step of food images, and the selection of three color channels can be defined.

$$
\begin{aligned}
& R^{\prime}=\frac{R}{255} \\
& G^{\prime}=\frac{G^{\prime}}{255} \\
& B^{\prime}=\frac{B}{255} \\
& C \max =\max \left(R^{\prime}, G^{\prime}, B^{\prime}\right) \\
& C \min =\min \left(R^{\prime}, G^{\prime}, B^{\prime}\right) \\
& \Delta=C \max -C \min \\
& H=\left\{\begin{array}{l}
\left\{0^{\circ},\right. \\
60^{\circ} \times\left(\frac{G^{\prime}-B^{\prime}}{\Delta} \bmod 6\right), \quad C \max =R^{\prime} \\
60^{\circ} \times\left(\frac{B^{\prime}-R^{\prime}}{\Delta}+2\right), \quad C \max =G^{\prime} \\
60^{\circ} \times\left(\frac{R^{\prime}-G^{\prime}}{\Delta}+4\right),
\end{array}\right. \\
& S=\left\{\begin{array}{c}
0, \quad \Delta=0 \\
\frac{\Delta}{1-|2 L-1|},
\end{array}\right. \\
& L=\frac{C \max +C \min }{2}=B^{\prime}
\end{aligned}
$$

In RGB to HSL conversion, first the RGB channel is normalized by dividing each of the channel with 255. After the RGB channel are normalized, the Cmax, Cmin, and delta are computed, with Cmax equals to the maximum value of each normalized RGB channel, Cmin equals to the minimum value of each normalized RGB channel, and delta equals to the result of $\mathrm{Cmax}$ subtracted by $\mathrm{Cmin}$. For the $L$ channel, the value equals to $\mathrm{Cmax}$ plus Cmin divided by 2 . For the $S$ channel, if delta equals 0 , then the value is 0 , else the value is the result of delta divided by 1 minus the absolute value of 2 times $L$ minus 1 . For the $H$ channel, if delta equals 0 , then the value is 0 . 
Else if Cmax equals to the normalized $\mathrm{R}$ channel, the value is normalized $G$ channel subtracted by is normalized $B$ channel, divided with delta, modulo by 6 , then multiplied with 60 degrees. Else if Cmax equals to the normalized $G$ channel, the value is a normalized $B$ channel subtracted by normalized $R$ channel, divided by delta, plus 2 , then multiplied by 60 degrees. Else if Cmax equals to the normalized $B$ channel, the value is a normalized $R$ channel subtracted by being normalized $G$ channel, divided by delta, plus 4 , then multiplied by 60 degrees.

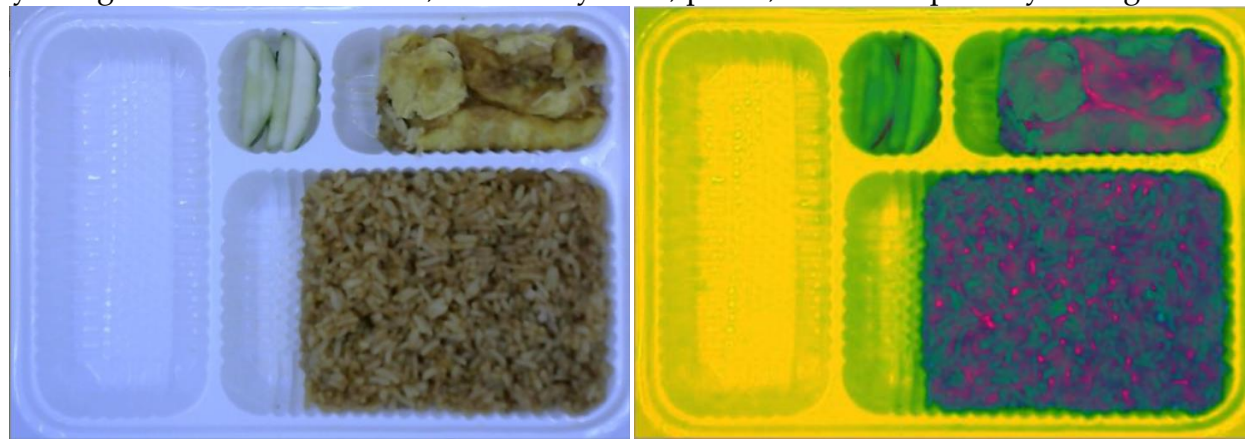

Figure 5. Image comparison between original image with HSL converted image

YUV

The YUV model consists of a luminance/brightness component $(Y)$ and two-color/chrominance content components $(U$ and $V$ ). Based on references $U$ of YUV color channel in image segmentation achieved above $90 \%$ when identifying medical plant leaf [20]. The formula for converting RGB to YUV is displayed in Equation 11.

$Y=0.299 \times R+0.587 \times G+0.114 \times B$

$U=0.493(B-Y)$

$V=0.877(R-Y)$

In RGB to YUV conversion, the $Y$ channel computed first, with $Y$ equals to the sum of each RGB channel multiplied by a constant for each channel. With $Y$ channel value, the other two channels can be calculated, with $U$ channel using $Y$ value to subtract from $B$ channel and $V$ channel using $Y$ value to subtract from $R$ channel, then both of them multiplied by a constant for each channel.
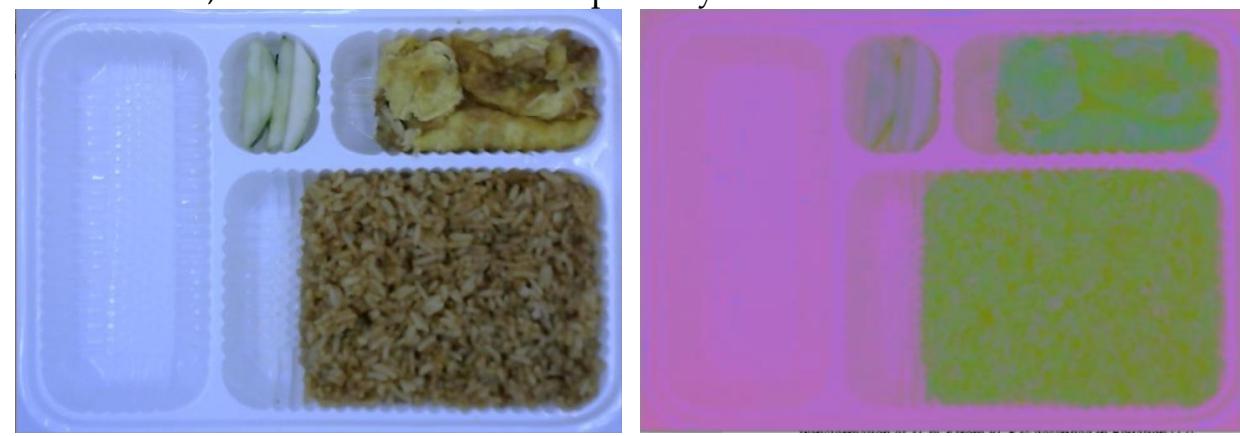

Figure 6. Image comparison between the original image with YUV converted image

\section{$\mathrm{YCbCr}$}

YCbCr contains three color channels, where $Y$ represents the color's luminosity, in which this channel is more sensitive to the human eye. Meanwhile, $\mathrm{Cb}$ and $\mathrm{Cr}$ are red and blue components. The color transformation of $\mathrm{YCbCr}$ from RGB is described in Equation 12.

$$
\left[\begin{array}{c}
Y \\
C_{b} \\
C_{r}
\end{array}\right]=\left[\begin{array}{ccc}
0.299 & 0.587 & 0.114 \\
-0.169 & -0.331 & 0.500 \\
0.500 & -0.419 & -0.081
\end{array}\right] \cdot\left[\begin{array}{l}
R \\
G \\
B
\end{array}\right]+\left[\begin{array}{c}
0 \\
128 \\
128
\end{array}\right]
$$
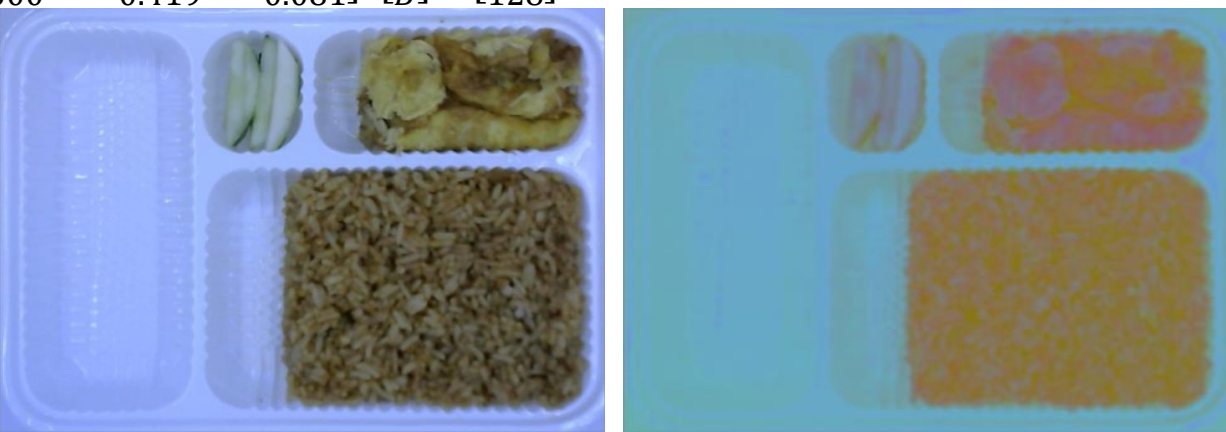

Figure 7. Image comparison between the original image with $\mathrm{YCbCr}$ converted image 
The color transformation from RGB to $\mathrm{YCbCr}$ computes each channel by using matrix multiplication with a constant matrix. After the matrix multiplication, a constant is added to each channel.

\subsection{Evaluation}

For testing, Ground truth images were used. The ground truth images were made from manually segmenting each image from the data. These manually segmented images were used as the perfect segmented image to evaluate and test the image for the metrics to test the segmented images' quality, Intersection Over Union (IOU), and Dice Score [15]. IOU is chosen based on the advantage in considering the proportion of the properly assigned pixels and penalizing false positive [15]. Dice score is also used as a metrics accompanying IOU for the reason that IOU and Dice's score is a great metrics to use, although not interchangeably [17]. The IOU and Dice Score calculation [21] as follows Equation 13 and Equation 14, respectively.

$I O U=\frac{I}{U}$
Dice $=\frac{2 I}{U+I}$

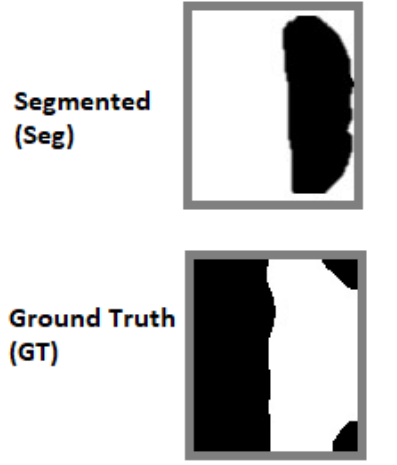

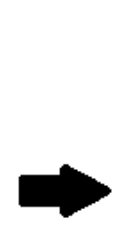

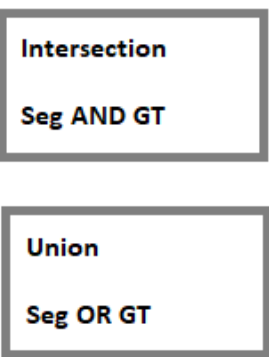

The $I$ and $U$ in these equations are intersection and union of the segmented image with the ground truth image. Intersection represents AND operation of segmented image and ground truth image, while union represents OR operation of segmented image and ground truth image. For the details on the calculation, in Figure 3 the calculation flow is visualized, which in both intersection or union was the sum of each pixel on segmented image and ground truth image. After intersection and union were calculated, each of them will be used on the calculation following Equation 13 and Equation 14.

\section{Results and Discussion}

The example results of the experiment are displayed in Table 1 . The first column of Table 1 is the original image, which is processed into segmentation in the different color channels. The ground truth is displayed in the middle column of Table 1, and the rest are graphs of IOU and Dice for color channels of color spaces. From those experimental results, it can be seen from the tren of IOU and Dice Score that $H$ of HLS color channel almost stable for various images to segment the object. Also, $A$ of LAB color channel steady for the whole image input. Both HLS-H and LAB-A have IOU and Dice Score above $90 \%$ on average compared to the color channels and color spaces. It means that segmentation by using those color channels is close to the ground truth image.

The problem is when the compartment of the tray box also has a blank (no.-food) area. The segmented area should be black in the whole area. However, the shadow from the edge of the tray box, the textured background of tray box and reflection, influences the segmented image quality. So, the IOU and Dice score from the blank compartment may far from the expected value to get sufficient segmented area of food image, as stated in Table 3. In Table 3, Image 1 reaches a higher score of IOU and Dice comparing to Image 2 . The broader area of blank compartment more the problem of segmentation. The smaller area of blank compartment uses $H$ of HLS can cover the problem, which can be proven by having IOU and Dice Score, 0.96 and 0.98 , respectively.

Meanwhile, applying $A$ of LAB in the broader area is more suitable, which represents from IOU and Dice Score, 0.85 and 0.92 consecutively. To ensure that the IOU and Dice Score can serve the quality of image segmentation, Table 2 depicts the information based on the visualization of how each color 
L. Maulana et al./Register 6 (2) 2020 pp. 141-151

ISSN 2502-3357 (Online)

channels and color spaces cope with the problem. In Image 1, the segmented area of using HLS-H achieves the best among the whole color channels and color spaces, so for LAB-A in Image 2.

Table 1. The IOU and Dice Score of given food image

\begin{tabular}{ll} 
Original Image & Ground truth \\
\hline
\end{tabular}
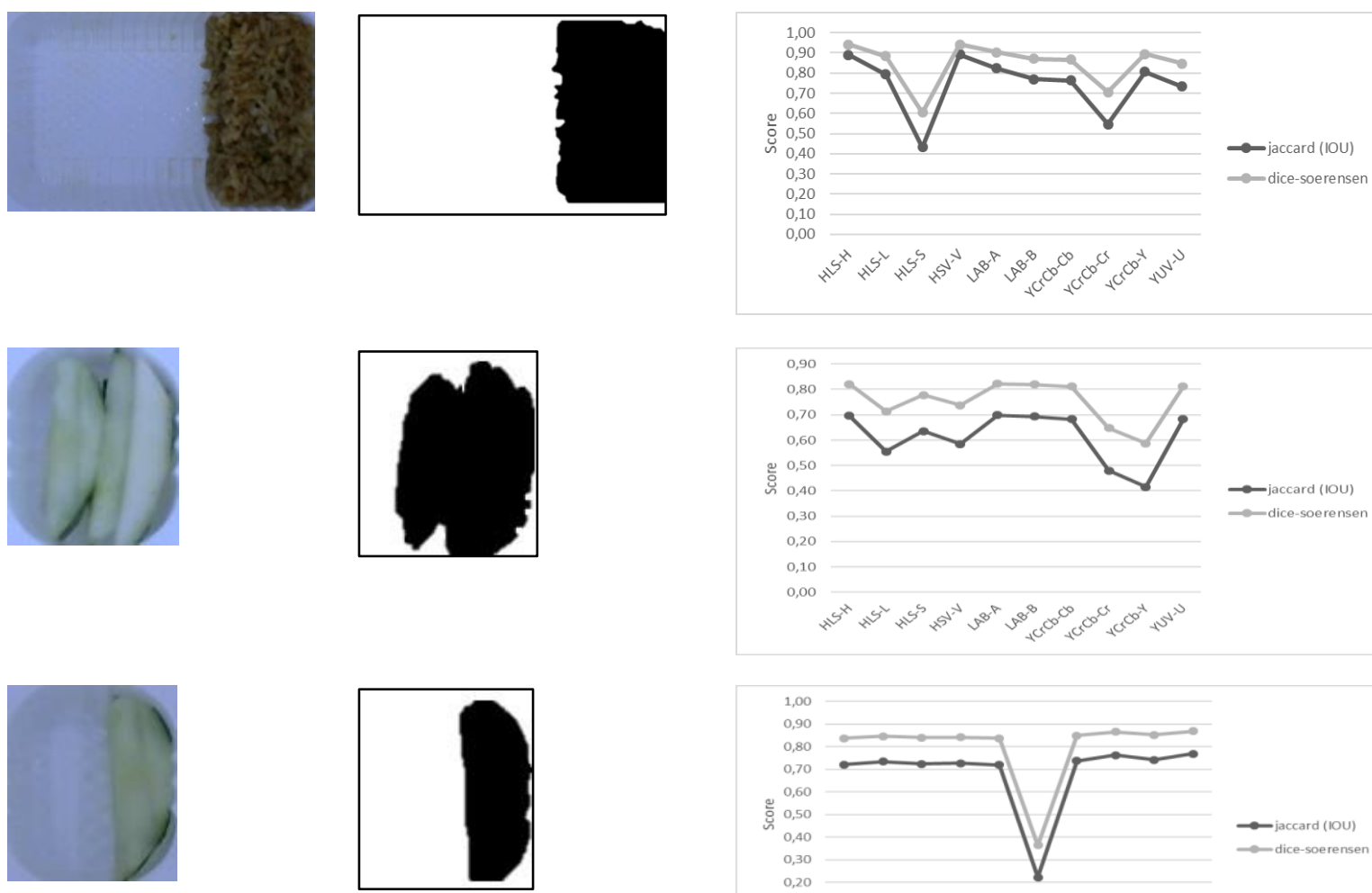

Segmented image score in each color channel
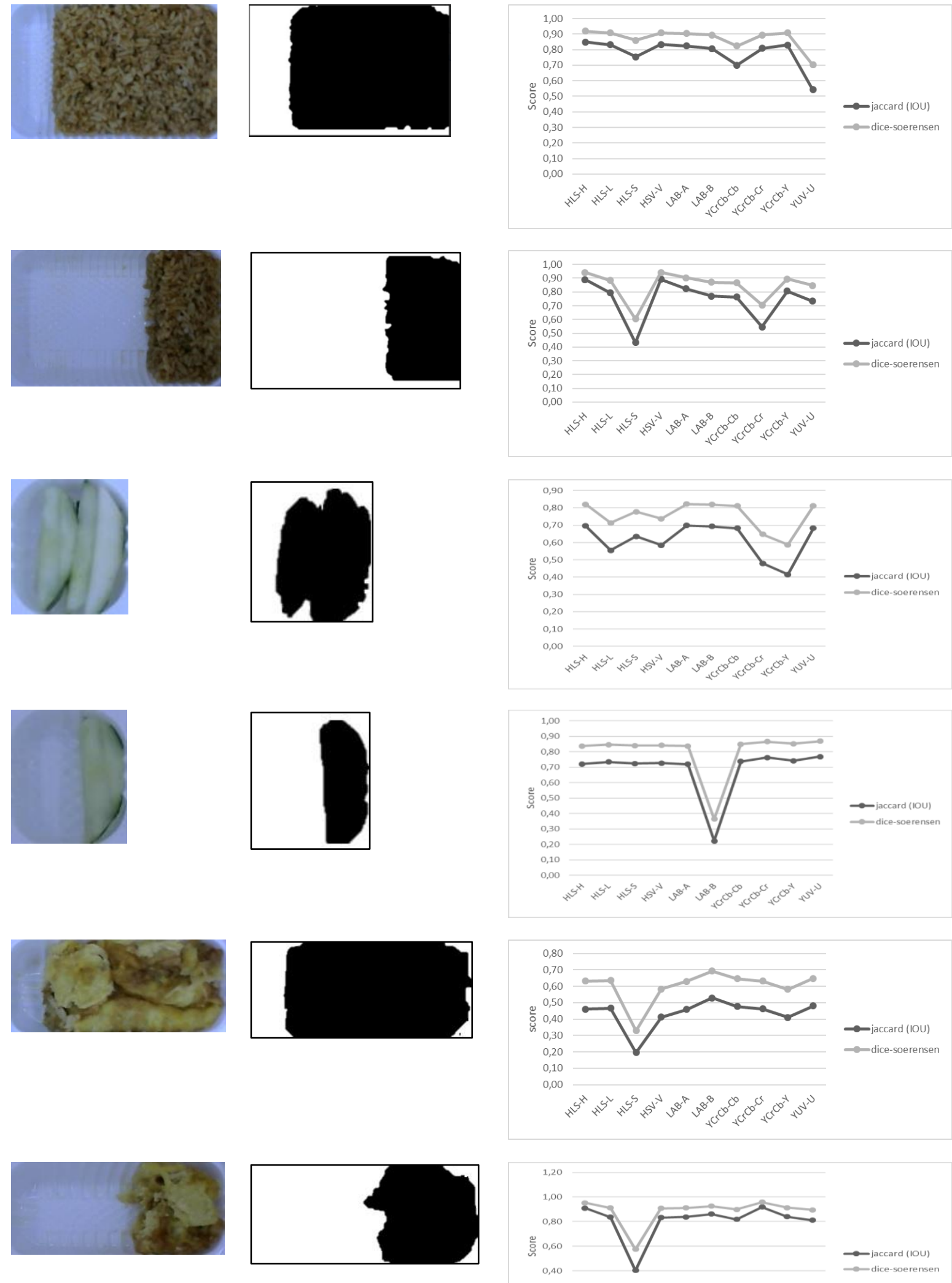

$\rightarrow$ - jaccard (IOU)
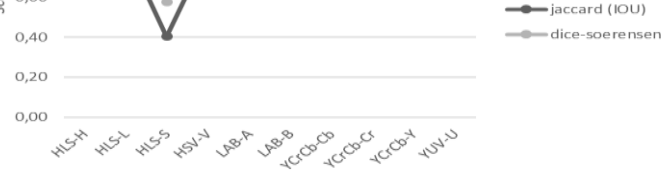
Table 2. The segmented result from a blank compartment

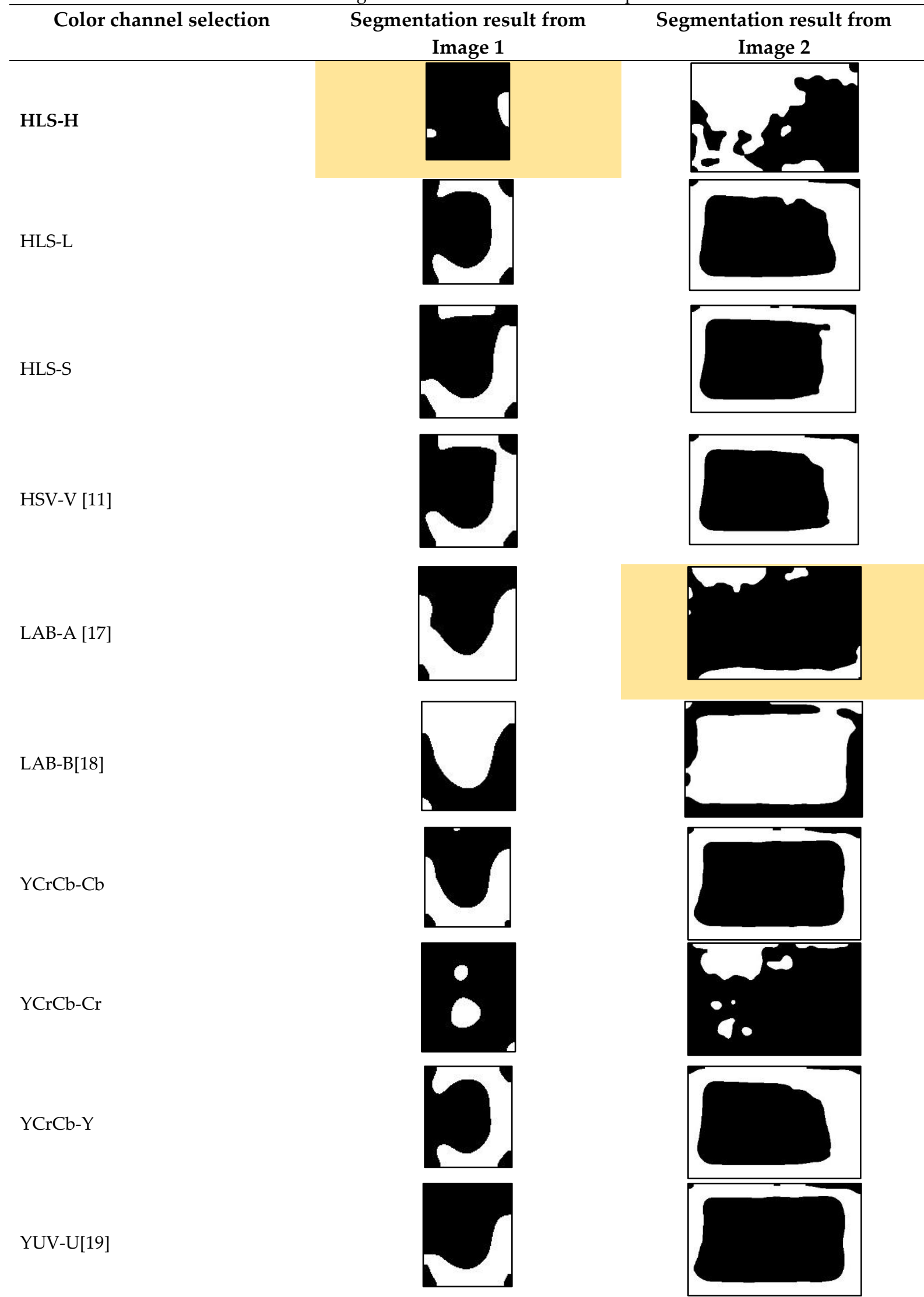

The scoring of the total of combining IOU and Dice can be seen in Table 4 . The scoring calculated with each image's IOU and Dice Score, where the image's best and worst score be counted with the best score given +1 score and worst score -1 . The score will be accumulated for each color channel, with the highest score declared the best color channel. 
Table 3. The problem in the blank compartment

\begin{tabular}{|c|c|c|c|c|}
\hline $\begin{array}{l}\text { Blank } \\
\text { compartment } \\
\text { image }\end{array}$ & & & & \\
\hline Color Channel & IOU & Dice & IOU & Dice \\
\hline HLS-H & 0,96 & 0,98 & 0,50 & 0,67 \\
\hline HLS-L & 0,62 & 0,77 & 0,55 & 0,71 \\
\hline HLS-S & 0,64 & 0,78 & 0,56 & 0,72 \\
\hline HSV-V [11] & 0,63 & 0,77 & 0,55 & 0,71 \\
\hline LAB-A [17] & 0,57 & 0,73 & 0,85 & 0,92 \\
\hline LAB-B[18] & 0,60 & 0,75 & 0,72 & 0,84 \\
\hline $\mathrm{YCrCb}-\mathrm{Cb}$ & 0,62 & 0,77 & 0,66 & 0,80 \\
\hline $\mathrm{YCrCb}-\mathrm{Cr}$ & 0,12 & 0,21 & 0,16 & 0,28 \\
\hline YCrCb-Y & 0,60 & 0,75 & 0,54 & 0,70 \\
\hline YUV-U[19] & 0,73 & 0,84 & 0,66 & 0,79 \\
\hline
\end{tabular}

From the results in Table 4, the LAB-A color channel and HSL-H color channel achieve the highest score at 5 . The lowest score came from the HLS-S color channel with -5 . The surprising part came from the LAB-B color channel, which has scoring second-worst at -4 . The $\mathrm{YCrCb}-\mathrm{Cb}$ color channel stands as neutral with 0 scores like $\mathrm{YCrCb}-\mathrm{Cr}$ and YUV-U color channel.

\begin{tabular}{|c|c|c|c|c|c|c|c|c|c|c|}
\hline & $\begin{array}{c}\text { LAB-A } \\
{[17]}\end{array}$ & $\begin{array}{c}\text { LAB-B } \\
{[18]}\end{array}$ & $\begin{array}{c}\text { HSV- V } \\
{[11]} \\
\end{array}$ & $\begin{array}{l}\text { YUV - } \\
\text { U[19] }\end{array}$ & $\begin{array}{c}\text { YCrCb } \\
\text { Y }\end{array}$ & YCrCb Cb & YCrCb Cr & HLS H & HLS S & HLS L \\
\hline Best & 5 & 1 & 2 & 2 & 0 & 0 & 2 & 8 & 0 & 0 \\
\hline Worst & 0 & 5 & 1 & 2 & 1 & 0 & 2 & 3 & 5 & 1 \\
\hline Total & 5 & -4 & 1 & 0 & -1 & 0 & 0 & 5 & -5 & -1 \\
\hline
\end{tabular}

From the result, the HSL-H color channel seems to hold more best scores than the LAB-A color channel. However, the LAB-A color channel does not suffer any worst score whatsoever. Meanwhile, analyzed more further, when HSL-H color channel score is at the worst, LAB-A color channel is more likely to have a better score or even the best score. Also, when the HSL-H color channel is on top, the score of A color channel is still passable, sometime placed second. With the domination of HSL-H and LAB-A color channels, Hue and Red-Green color range are meaningful representations of the images. The unfortunate result of the HSL-S color channel shows that Saturation and lighting effect were not suitable for representing the images. The LAB-B color channel result also shows that previous research using the LAB-B color channel is not optimal for these images, and the Blue-Yellow color range is not optimal.

\section{Conclusions}

In conclusion, $A$ color channel was proven to be the best color channel to be used in Food Leftover Estimation, although the $H$ color channel yields better results than $A$ color channel. $A$ color channel is more stable than the $H$ color channel, with $A$ color channel scoring five best and 0 worst and $H$ color channel scoring eight best and three worst.

For future research, different color channels can be used. Also, different metrics can be used to determine the quality of the segmentation. The quantity of the data also can be added, regarding the small size of the data with five images yielding only 200 data, as a response to the observed limitation. Moreover, the implications of the research must also be included. Combining with other image enhancement techniques is considering to have a better result of the segmentation of food images.

\section{Acknowledgement}

The authors would like to acknowledge the support of the Computer Vision Laboratory to use the laboratory room to do this research. Besides, the authors thank the Faculty of Computer Science, who fully supports this research and research center of the University of Brawijaya (LP3M) to support this collaboration of continuous research. The authors also regard for Agricultural Technology Department Program to help of collecting the dataset.

\section{References}


[1] J. Dehais, M. Anthimopoulos and S. Mougiakakou, "Food Image Segmentation for Dietary Assessment," in MADiMa '16: Proceedings of the 2nd International Workshop on Multimedia Assisted Dietary Management, Amsterdam, Netherlands, 2016.

[2] G. Ciocca, P. Napoletano and R. Schettini, "Food Recognition and Leftover Estimation for Daily Diet Monitoring," in ICIAP 2015: New Trends in Image Analysis and Processing, Cham, 2015.

[3] S. Mezgec, T. Eftimov, T. Bucher and B. K. Seljak, "Mixed deep learning and natural language processing method for fake-food image recognition and standardization to help automated dietary assessment," Public Health Nutrition, vol. 22, no. 7, p. 1193-1202, 2018.

[4] Y. He, C. Xu, N. Khanna, C. J. Boushey and E. J. Delp, "Food image analysis: Segmentation, identification and weight estimation," in IEEE International Conference on Multimedia and Expo (ICME), San Jose, CA, 2013.

[5] Y. A. Sari, R. K. Dewi, J. M. Maligan, L. Maulana and S. Adinugroho, "Automatic Leftover Weight Prediction in Tray Box Using Improved Image Segmentation Color Lighting Component," Journal of Southwest Jiaotong University, vol. 55, no. 1, 2020.

[6] S. Inunganbi, A. Seal and P. Khanna, "Classification of Food Images through Interactive Image Segmentation," in Asian Conference on Intelligent Information and Database Systems, Cham, 2018.

[7] Y. A. Sari, F. Utaminingrum, S. Adinugroho, R. K. Dewi, P. P. Adikara, R. C. Wihandika, S. Mutrofin and A. Izzah, "Indonesian Traditional Food Image Identification using Random Forest Classifier based on Color and Texture Features," in International Conference on Sustainable Information Engineering and Technology (SIET), Lombok, Indonesia, 2019.

[8] G. Ciocca, P. Napoletano and R. Schettini, "Learning CNN-based Features for Retrieval of Food Images," in International Conference on Image Analysis and Processing, Cham, 2017.

[9] K. Aizawa, K. Maeda, M. Ogawa, Y. Sato, M. Kasamatsu, K. Waki and H. Takimoto, "Comparative Study of the Routine Daily Usability of FoodLog: A Smartphonebased Food Recording Tool Assisted by Image Retrieval," Journal of Diabetes Science and Technology, vol. 8, no. 2, p. 203-208, 2014.

[10] A. F. Ahsani, Y. A. Sari and P. P. Adikara, "Food Image Retrieval with Gray Level Co-Occurrence Matrix Texture Feature and CIE L*a*b* Color Moments Feature," in International Conference on Sustainable Information Engineering and Technology (SIET), Lombok, Indonesia, 2019.

[11] Y. A. Sari, J. M. Maligan and Y. G. B. Sigit Adinugroho, "Multiple Food or Non-Food Detection in Single Tray Box Image using Fraction of Pixel Segmentation for Developing Smart NutritionBox Prototype," International Journal of Innovative Technology and Exploring Engineering (IJITEE), vol. 9, no. 3S, 2020.

[12] Y. A. Sari, R. K. Dewi, J. M. Maligan, A. S. Ananta and S. Adinugroho, "Automatic Food Leftover Estimation in Tray Box Using Image Segmentation," in International Conference on Sustainable Information Engineering and Technology (SIET), Lombok, Indonesia, 2019.

[13] L. Busin, N. Vandenbroucke and L. Macaire, "Color Spaces and Image Segmentation," in Advances in Imaging And Electron Physics, Elsevier, 2008.

[14] J. Diaz-Cely, C. Arce-Lopera, J. C. Mena and L. Quintero, "The Effect of Color Channel Representations on the Transferability of Convolutional Neural Networks," in Science and Information Conference, Cham, 2020.

[15] V. K. Singh, H. A. Rashwan, M. Abdel-Nasser, M. M. K. Sarker, F. Akram, N. Pandey, S. Romani and D. Puig, "An Efficient Solution for Breast Tumor Segmentation and Classification in Ultrasound Images Using Deep Adversarial Learning," arXiv, 2019.

[16] K. J. Pfisterer, R. Amelard, A. G. Chung, B. Syrnyk, A. MacLean and A. Wong, "Fully-Automatic Semantic Segmentation for Food Intake Tracking in Long-Term Care Homes," arXiv, 2019.

[17] T. S. K. M. Rabie, "Implementation of Some Similarity Coefficients in Conjunction With Multiple Upgma and Neighbor-Joining Algorithm," Egypt. Poult. Sci., vol. 30, no. 2, pp. 607-621. 
[18] P. Harnis, Y. A. Sari and M. A. Rahman, "Segmentasi Citra Kue Tradisional menggunakan Otsu Thresholding pada Ruang Warna CIE LAB," Jurnal Pengembangan Teknologi Informasi dan Ilmu Komputer, vol. 3, no. 7, pp. 6799-6808, 2019.

[19] F. Y. Nabella, Y. A. Sari and R. C. Wihandika, "Seleksi Fitur Information Gain Pada Klasifikasi Citra Makanan Menggunakan Hue Saturation Value dan Gray Level Co-Occurrence Matrix," Jurnal Pengembangan Teknologi Informasi dan Ilmu Komputer, vol. 3, no. 2, pp. 1892-1900, 2019.

[20] C. H. Arun and D. C. Durairaj, "Identifying Medicinal Plant Leaves Using Textures and Optimal Colour Spaces Channel," Jurnal Ilmu Komputer dan Informasi, vol. 10, no. 1, pp. 19-28, 2017.

[21] A. Sevastopolsky, "Optic Disc and Cup Segmentation Methods for Glaucoma Detection with Modification of U-Net Convolutional Neural Network," Pattern Recognition and Image Analysis, vol. 27, p. 618-624, 2017. 